AperTO - Archivio Istituzionale Open Access dell'Università di Torino

\title{
Photochemical stability and reactivity of Graphene Oxide
}

\section{This is the author's manuscript}

Original Citation:

Availability:

This version is available http://hdl.handle.net/2318/1508059

since 2016-06-28T14:37:14Z

Published version:

DOI:10.1007/s10853-014-8791-1

Terms of use:

Open Access

Anyone can freely access the full text of works made available as "Open Access". Works made available under a Creative Commons license can be used according to the terms and conditions of said license. Use of all other works requires consent of the right holder (author or publisher) if not exempted from copyright protection by the applicable law. 


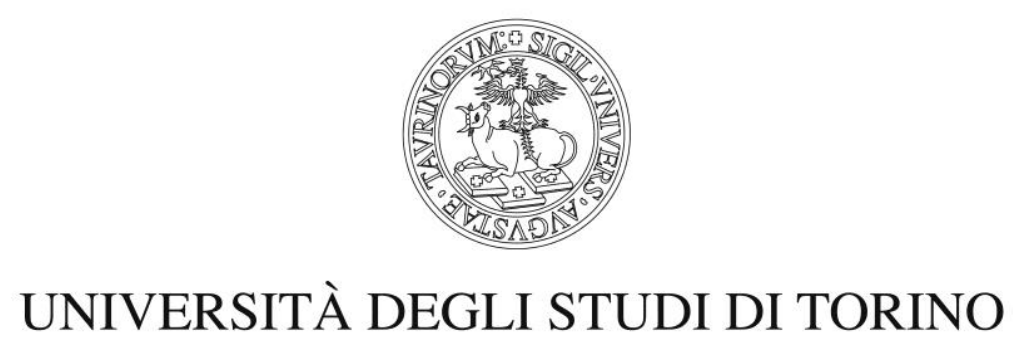

The final publication is available at Springer via http://dx.doi.org/10.1007/s10853-014-8791-1 


\title{
Photochemical stability and reactivity of Graphene Oxide
}

\author{
M. Minella, M. Demontis, M. Sarro, F. Sordello, P. Calza*, C. Minero
}

Department of Chemistry and NIS Center, University of Torino, Via P. Giuria 5,

Torino 10125, Italy http://www.environmentalchemistry.unito.it.

* Corresponding author. Fax +39-011-6705242; E-mail: paola.calza.@unito.it.

E-mail:

marco.minella@unito.it

mariangela.demontis@studenti.unito.it

marco.sarro@unito.it

fabrizio.sordello@unito.it

paola.calza@unito.it

claudio.minero@unito.it 


\section{Abstract}

The photoreactivity of graphene oxide (GO) suspensions was investigated with a double aim: $i$ ) to give insights into the previously reported photo-reduction process, which allows a partial elimination of the oxygen-containing groups from the 2D graphitic structure; $i$ ) to explore the possible use of GO as photo-activator able to promote the photo-transformation/abatement of organic molecules. To reach these goals and clarify some peculiar aspects of the photochemistry of GO till now obscure or confuse, we synthesized and characterized stable GO suspensions which were then subjected to UV-Vis irradiation for prolonged times. GO underwent partial photoreduction with the release of gaseous molecules and soluble organic species (e.g. carboxylic acid). The mechanisms of photo-reduction occurring under air or $\mathrm{N}_{2}$ are different, as assessed by the release in solution of diverse soluble molecules. In the presence of oxygen, at long irradiation time, a complete solubilization of the graphenic structures was observed. No difference in the nature and amount of released gases (principally $\mathrm{CO}_{2}$ and $\mathrm{CO}$ ) was observed in the oxic or anoxic conditions. The possible use of GO as photo-activator was evaluated by using phenol as probe molecule. GO revealed a double role of photo-activator and reagent in phenol degradation, as competition was assessed between GO self-transformation/reduction and phenol degradation. At prolonged irradiation time a marked reactivity of the photoformed species was observed and the complete degradation was achieved for both organic small molecules formed from GO and the phenol added as probe molecule.

Keywords: graphene oxide, photochemical stability, photo-activated degradation, advanced oxidation processes 


\section{Introduction}

The current syntheses of graphene oxide (GO) from graphite are based on different approaches, e.g. the acid oxidation of Brodie [1] and Staudenmaier [2], which exploits the combination of $\mathrm{KClO}_{3}$ and $\mathrm{HNO}_{3}$, or the Hummers' method [3], in which the active species acting as oxidant for the graphite is $\mathrm{Mn}_{2} \mathrm{O}_{7}$, formed by the reaction of $\mathrm{KMnO}_{4}$ with $\mathrm{H}_{2} \mathrm{SO}_{4}$. The oxidation produces a material containing oxygenated groups able to destroy the $\pi$ configuration; consequently, the produced graphene oxide will behave as an insulator. The structure of graphene oxide has been studied by several research groups [4] and the currently accepted model has been proposed by LerfKlinowsky, in which a non-stoichiometric amorphous structure is proposed [5] and the basal plane of graphite oxidized is decorated with epoxy and hydroxyl groups; carbonyl and carboxyl groups are also present along the edges of planes.

GO can be reduced to form the so-called reduced graphene oxide (rGO). Even if numerous methods for GO reduction were proposed, e.g. via thermal or photothermal processes, recently attention has been focused on the use of a photochemical process, as it does not involve the use of potentially toxic reagents and exploits less restrictive operating conditions. The photoreduction of GO using only the UV light leads to a decrease of the electrical resistance and to an increased absorption in the visible spectral region, with a change in color of the suspension from light brown to dark brown, almost black, which can be interpreted as the renewal of conjugation.

Graphene oxide and its controlled reduction allowed progressive tuning of the optical gap from 3.5 $\mathrm{eV}$ down to $1 \mathrm{eV}$, and XPS spectra show contemporaneous increase in the $\mathrm{C} / \mathrm{O}$ ratio [6]; for such, this material could behave as a photocatalyst. [7] At present, the ability of GO to act as photosensitizer has not yet been studied, while several approaches were employed to prepare hybrid materials containing GO (or rGO). Williams et al. [8] synthesized a hybrid $\mathrm{rGO}-\mathrm{TiO}_{2}$ material by irradiating a suspension containing $\mathrm{TiO}_{2}$ nanoparticles and GO. The use of $\mathrm{rGO}$ was proposed by several groups for the preparation of highly photoactive composite materials based on titanium dioxide for the catalytic oxidation of aqueous organic pollutants [9-14], in order to exploit the high charge mobility of graphene and to maximize the photocatalytic efficiency by hindering the process of charge recombination.

The aim of this work was to study the stability and transformation of GO during the photochemical reduction and the contemporaneous assessment of its ability to act as a photosensitizer toward the abatement of micropollutants. For the latter aspect, phenol was chosen as a target molecule. The study 
of the photostability of the material was evaluated with a combined evaluation of what occurs on the aqueous suspension and in gas phase. This goal was achieved by using ATR-FTIR, UV-Vis spectrophotometry, fluorescence spectroscopy, ion chromatography, gas chromatography and TOC analyses.

\section{Experimental section}

\section{Materials}

Phenol was purchased from Aldrich, acetonitrile (AC0331 Supergradient HPLC grade eluent) from Scharlau, graphite natural powder (briquetting grade, $\approx 100$ mesh, 99.9995\%) from Alfa Aesar, $\mathrm{H}_{3} \mathrm{PO}_{4}(85 \%), \mathrm{H}_{2} \mathrm{SO}_{4}(96 \%), \mathrm{KMnO}_{4}(>99 \%), \mathrm{HCl}(37 \%)$, methanol gradient grade from Carlo Erba. Zero-grade air for TOC analysis was bought from Sapio (Turin, Italy). All the compounds were used as received without any further purification step. All aqueous solutions were prepared with ultrapure water Millipore Milli- $\mathrm{Q}^{\mathrm{TM}}(\mathrm{TOC}<2 \mathrm{ppb}$, conductivity $\geq 18 \mathrm{M} \Omega \mathrm{cm})$.

\section{Methods}

\section{Synthesis of GO}

GO for photochemical experiments was produced by means of chemical exfoliation of oxidized graphite according to the modified Hummers and Staudemaier's method [2,3] proposed by Huang et al. [15]. The details of the synthesis are here briefly summarized. A suspension of $500 \mathrm{mg}$ of graphite in $\mathrm{H}_{2} \mathrm{SO}_{4}, \mathrm{H}_{3} \mathrm{PO}_{4}$ and $\mathrm{KMnO}_{4}(27.3 \mathrm{ml}, 3.30 \mathrm{~mL}$ and $2.8 \mathrm{~g}$, respectively) was prepared and left under magnetic stirring for different times (e.g. 1, 2 and 3 days), after which $120 \mathrm{~mL}$ of water were added, the viscous suspension cooled with ice, and the non reacted $\mathrm{MnO}_{4}{ }^{-}$titrated with $\mathrm{H}_{2} \mathrm{O}_{2}$. The pale yellow graphite oxide obtained was separated by means of centrifugation, three times washed with $\mathrm{HCl} 1 \mathrm{M}$ and then with water. During the washing procedure graphite oxide underwent exfoliation and a viscous brown suspension of GO was produced. The GO suspensions obtained were purified by means of dialysis against water with Spectra/Por® Dialysis Membrane (MW cut off 6-8000 Da). The carbon loading of the obtained suspensions was evaluated by measuring the concentration of total organic carbon (TOC) in diluted GO suspensions. The size of the GO particles obtained after exfoliation at different oxidation times was evaluated measuring the hydrodynamic radius of the GO particles in diluted suspension with a Dynamic Light Scattering (ALV-NIBS with a He-Ne laser, $\lambda=632.8 \mathrm{~nm}$, and a correlator ALV-5000). The hydrodynamic 
radius of the GO particles was evaluated with the cumulant method and expressed as the average of at least three repetitions.

\section{Photochemical Experiments}

The irradiation tests of GO suspensions (to assess both the GO photostability and its photosensitized phenol degradation ability) were carried out in magnetic stirred Pyrex glass cells under a set of three TLK 40W/05 (UV-Vis) fluorescent lamps (Phillips, Eindhoven, Nederland) with emission spectrum centred at $360 \mathrm{~nm}$ (the irradiation experimental equipment and the emission spectra of the adopted irradiation system are reported in the Electronic Supplementary Material, hereafter SM). The UV integrated irradiance on the cells in the $290-400 \mathrm{~nm}$ range wavelengths was $24 \pm 1 \mathrm{~W} \mathrm{~m}^{-2}$ (measured with a CO.FO.MEGRA. (Milan, Italy) power-meter).

The GO photostability experiments were carried out on $10 \mathrm{~mL} \mathrm{GO}$ suspension. At zero time and after the desired irradiation time the suspensions were filtered on Millipore cellulose HAWP 0.45 $\mu \mathrm{m}$ membrane and the filtered clear solution or the filter-retained solid particles analyzed. The UVVis absorption spectra were recorded with a Varian CARY 100 Scan UV-Vis spectrophotometer, using Suprasil quartz cuvettes with a path length of $1 \mathrm{~cm}$. The fluorescence spectra (as Excitation Emission Matrixes, EEMs) were obtained with a Varian Cary Eclipse fluorescence spectrofluorimeter, adopting a $10 \mathrm{~nm}$ slit-width for both excitation and emission signals. The TOC concentration of the filtered suspensions was evaluated with a Shimadzu TOC-VCSH Total Organic Carbon Analyzer, equipped with an ASI-V autosampler and fed with zero-grade air, while the concentration of organic/inorganic anions with a Dionex DX 500 Ion Chromatograph, equipped with LC 30 chromatography oven, GP 40 gradient pump, Dionex Ion Pac AG9-HC 4-mm (10-32) guard column, Dionex Ion Pac AS9-HC 4-mm (10-32) anion exchange column, ASRS-ULTRA 4mm conductivity suppression unit, and ED 40 electrochemical detector (eluent $\mathrm{NaHCO}_{3} / \mathrm{K}_{2} \mathrm{CO}_{3}$ $4.5 \mathrm{mM} / 11 \mathrm{mM})$. The ATR-FTIR spectra of the solid particles collected on the filtering membrane were recorded with a Bruker IFS 28 (DTGS detector, $4 \mathrm{~cm}^{-1}$ resolution) by using a micro-ATR cell. The release of gas during irradiation was evaluated on more concentrated GO suspensions and under irradiation with a low pressure Hg vapor lamp (Sylvania H44GS-100, emission spectrum reported in Fig.1B-SM, UV irradiance reaching the sample $60 \mathrm{~W} \mathrm{~m}^{-2}$ ). The headspace was collected and analyzed with a micro-GC Agilent 490 Micro GC equipped with two different modules for the analysis of $\mathrm{CO}$ and other permanent gases (Molsieve 5A) and $\mathrm{CO}_{2}, \mathrm{CH}_{4}$ and hydrocarbons till $\mathrm{C6}$ (CP PoraPLOT U). 
We chose phenol as a target molecule to investigate the photosensitizer ability of the material as phenol does not absorb under the adopted condition of irradiation. We irradiated $5 \mathrm{~mL}$ of samples running all the experiments without modifying the solution $\mathrm{pH}$. The disappearance of phenol was followed as a function of irradiation time using a high pressure liquid chromatography system (Merck-Hitachi L-6200 pumps), equipped with a Rheodyne injector, an RP C18 column (Lichrochart, Merck, $12.5 \mathrm{~cm}$ x $0.4 \mathrm{~cm}, 5 \mu \mathrm{m}$ packing) and a UV-Vis detector (Merck Hitachi L4200) set at $220 \mathrm{~nm}$. We eluted the samples with acetonitrile and phosphate buffer $(0.01 \mathrm{M})$ at $\mathrm{pH}$ $2.8(40: 60 \% \mathrm{v} / \mathrm{v})$ at a flow rate of $1 \mathrm{~mL} \mathrm{~min}^{-1}$. We ran all experiments in triplicates, and we report in the paper the average results.

\section{Results}

\section{GO suspension characterization (different oxidation time)}

Different analytical approaches (UV-Vis absorption and fluorescence spectroscopy, ATR-FTIR spectroscopy and Dynamic Laser Scattering) have been chosen to characterize the suspensions of pristine GO obtained at different oxidation times with the aim of estimating the degree of GO oxidation and the size of the particles/aggregates obtained.

Fig.1 shows the UV-Vis absorption spectra normalized for the TOC concentration of the GO suspensions obtained after 1, 2 and 3 days of oxidation. All the spectra are dominated by the 230 $\mathrm{nm}$ absorption related to the $\pi \rightarrow \pi^{*}$ transition of the $\mathrm{C}=\mathrm{C}$ bonding [15-17]. The main absorption peaks show a shoulder at $300 \mathrm{~nm}$ due to the $\mathrm{n} \rightarrow \pi^{*}$ transition of the carbonyl groups $[18,19]$ generated during the chemical oxidation of the graphite planes. The ratio between the normalized absorption at 230 and $300 \mathrm{~nm}$ is informative of the oxidation degree of the carbonaceous structure. In our case the ratio is 3.0, 3.6 and 3.7 for the sample obtained after 1, 2 and 3 days of oxidation, respectively. The increment is related to the increase of the oxidation degree of the basal plane of graphene with an associated increment of the isolated aromatic ring concentration and consequently of the absorbance at $230 \mathrm{~nm}$. The ratio increases with the increment of the oxidation time, but this value does not change dramatically as a consequence of the high degree of oxidation already reached after the first day of oxidation. The obtained ratios (>2) are very similar to those reported by Huang et al. [15] for GO samples with significant higher lateral dimensions.

On the basis of the suggestions reported by Shang et al [20] the Excitation Emission Matrixes (EEMs) of diluted suspensions of GO obtained at different oxidation time (1, 2 and 3 days) were 
recorded in the 300-650 $\mathrm{nm}$ and 300-800 $\mathrm{nm}$ ranges for excitation and emission, respectively. Fig.2 shows the EEMs recorded on the suspensions of GO obtained at different reaction times. In each case a broad emission peak centered at excitation/emission coordinates $350 / 540 \mathrm{~nm}$ is manifest with progressive decrement of the emission intensity with the increase of the oxidation degree of the graphene planes.

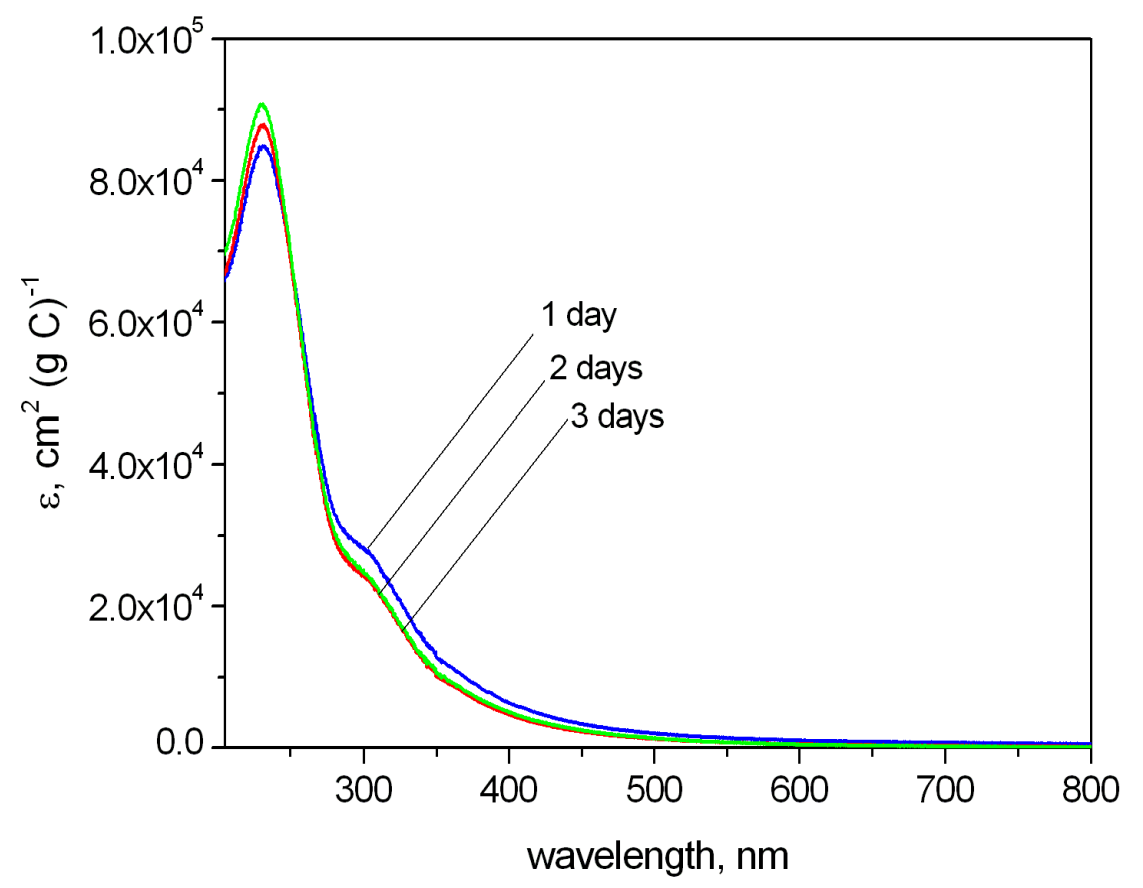

Fig.1 UV-Vis spectra, expressed as absorbance coefficient, of the GO suspensions obtained after 1, 2 and 3 days of oxidation.

The fluorescence of GO is a complex and already faced up issue [20-22]. It is not here the place for an in-depth review and analysis of the quantum mechanical processes at the basis of the GO emission. The emission seems to be related to a recombination process between excited electrons that can recombine in a process similar to the conduction/valence band electron recombination apparent in direct semiconductors. Note that perfect 2D graphene is a zero band gap material [23], while the introduction of doping elements or covalent functionalization (e.g. oxidized moieties such as in GO) generates a band gap, opening its applicability in functional nanoelectronic devices. $[24,25]$ The quenching of the fluorescence emission with the increment of the oxidation degree is related with the increase of the density of localized states that, as for the semiconductor, can force the dissipation energy processes toward non radiative paths (e.g. vibrational relaxation). From a molecular point of view, the decrement of the fluorescence signal intensity with the increment of the oxidation time can be easily related to the decrement of the aromaticity that give more flexibility 
to the 2D planes and consequently increases the possibility to dissipate energy with vibrational modes quenching the fluorescence emission.
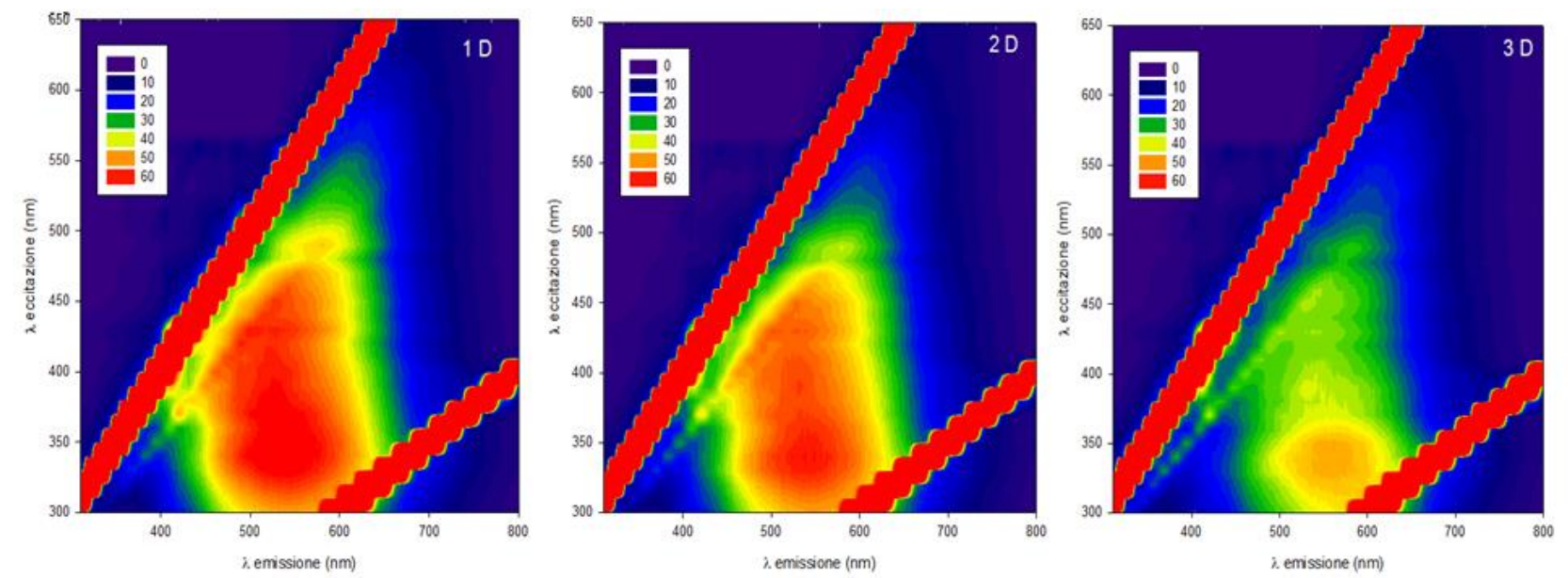

Fig.2 Excitation Emission fluorescence matrixes (EEMs) of the suspension of GO obtained, from left to right, after 1, 2 and 3 days of oxidation. The maximum of the fluorescence peak is in all the cases at $340 / 540 \mathrm{~nm}$. The linear elements in the EEMs are the first and second harmonic of the Rayleigh-Tyndall scattering $\left(\lambda^{\mathrm{ex}}=\lambda^{\mathrm{em}}\right.$ and $\lambda^{\mathrm{ex}}=2 \lambda^{\mathrm{em}}$, respectively) and the Raman signal of the water.

The qualitative and semi-quantitative study of the nature and concentration of oxidized moieties in the structure of the synthesized GO was carried out recording the ATR-FTIR spectra of GO collected on $0.45 \mu \mathrm{m}$ filtering membrane (note that the thickness of the GO analyzed films masked totally the FTIR signals of the membrane). Fig.3 shows the recorded spectra in which the peculiar vibrational signals of oxygen-containing groups typical of GO structures are manifest [26-28]. A summary of the recorded peak signals and their attribution are summarized in Table 1-SM. The nature of the oxidized moieties on the GO structure does not change with the increment of the oxidation time, while the relative importance of some peaks with respect to the intensity of the 1623 $\mathrm{cm}^{-1}$ signal (vibrational mode of $\mathrm{sp}^{2}$ carbon atoms in aromatic rings) varies. Chiefly, a slight increment of the ratio between the absorbance of the peaks at 1350, 1220 and $1040 \mathrm{~cm}^{-1}$ and that at $1623 \mathrm{~cm}^{-1}$ was observed as a consequence of the increment of the oxygen-containing groups concentration in the GO structures with the increment of the reaction time. 


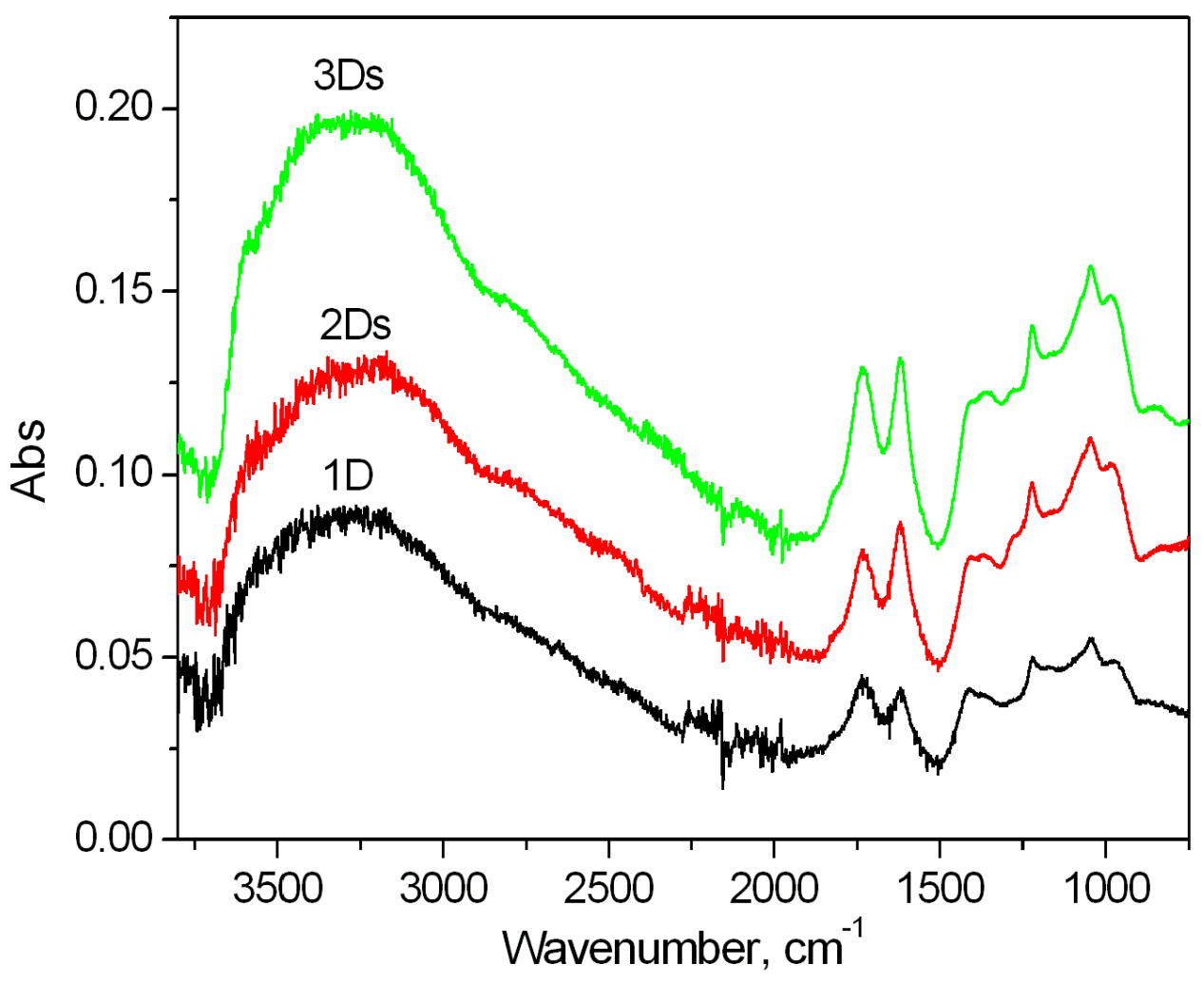

Fig.3 ATR-FTIR spectra of the GO obtained at 1, 2 and 3 days of oxidation. The GO suspensions were filtered on 0.45 $\mu \mathrm{m}$ filters and the spectra of the surface of the filters recorded.

The size of the GO particles in suspension obtained at the end of the oxidation (for different times) and exfoliation process were evaluated with Dynamic Light Scattering (DLS). This technique allows the simple, quick and cheap determination of the hydrodynamic radius $\left(r_{h y d}\right)$ of single particles (or aggregates). Fig.4 shows the evolution of the $r_{\text {hyd }}$ as a function of the oxidation time with a monotonic increment of the particle size: $214 \pm 37,328 \pm 60$ and $496 \pm 69 \mathrm{~nm}$, after 1,2 and 3 days of oxidation, respectively. These data are in good agreement with those reported by Huang and co-workers [15] who observed an increment of the lateral dimension of GO produced by means of the classic Hummers' method not only with the increment of the oxidation time, but also increasing the concentration of $\mathrm{KMnO}_{4}$ in the reaction media. The data reported in Fig. 4 can be explained in the light of the spectroscopic data reported above concerning the increment of the oxidized moieties on the graphene basal planes with the increment of the reaction time. This decreases the attractive interactions between the contiguous carbonaceous planes diminishing their fragmentation during the exfoliation procedure. The pristine GO obtained at different oxidation times showed an increment in the concentration of oxygen-containing groups, even if a high oxidation degree has been already obtained after the first day of oxidation. A further oxidation allows an increment of the concentration of the oxidized moieties which favors an easier exfoliation and consequently the 
production of GO particles with higher hydrodynamic radii. For the photochemical experiments we selected the suspension of GO obtained after 3 days of oxidation.

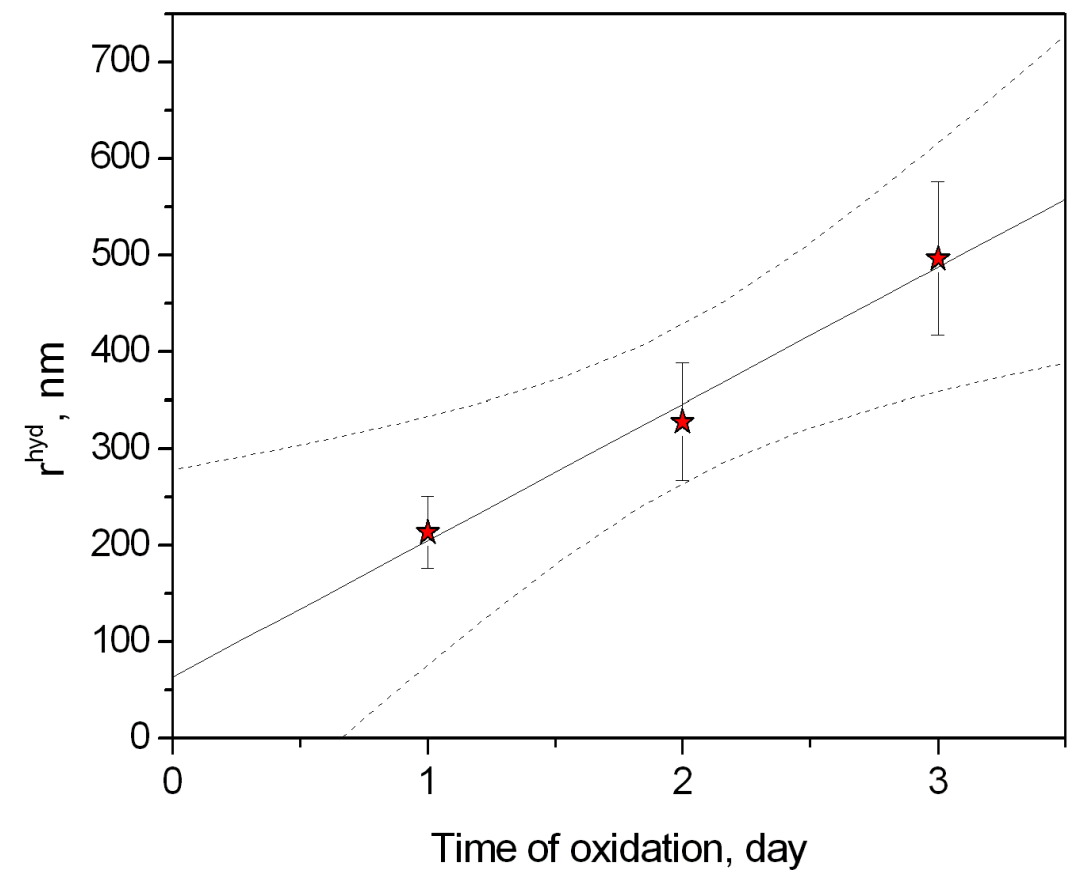

Fig.4 Hydrodynamic radius of the GO particles synthesized carrying out the oxidation of graphite flakes for different oxidation times.

\section{Photostability of GO in oxygenated and anoxic conditions}

The consequences of irradiation on the pristine GO suspensions were evaluated under both air and anoxic conditions. In the latter case the suspensions to be irradiated were previously purged with ultrapure nitrogen for 15 minutes and air seal caps were used. In the case of prolonged irradiation every two days the suspensions underwent a further purging step to clean the cells from oxygen which possibly can diffuse into the cells.

Fig.3-SM shows a selection of pictures of the cells containing GO $\left(30 \mathrm{mg} \mathrm{C} \mathrm{dm}^{-3}\right)$ after different irradiation times under air and under anoxic conditions. In both cases the progress of the photochemical processes is perceptible by the change of the suspension appearance. The pale yellow suspensions turned in both cases to brown (in the first day of irradiation) and then to grey/black suspensions where micrometric hydrophobic particles are present. The main difference between the two studied conditions was manifest at prolonged irradiation time: under oxygen the prolonged irradiation ( $t_{i r r}>14$ days) gave a complete dissolution of the hydrophobic particulates 
with formation of a transparent solution, while under anoxic conditions the photostability of the photoformed particles is higher and even after $t_{i r r}>14$ days the black particulate was present.

These first macroscopic data suggest that the irradiation activates a photo-reduction process both under air and under nitrogen which gives more hydrophobic particles which aggregate in water forming unstable colloids of reduced GO (rGO). Further evidence of the partially reduction of GO was obtained from the ATR-FTIR spectra, acquired on GO particulates obtained after irradiation, although with some difficulties, vide infra, and by XPS measures performed on pristine material and on GO after 1 day of irradiation, where evidences a decrease in the $\mathrm{C}=\mathrm{O} / \mathrm{O}-\mathrm{C}-\mathrm{O}$ content (see Fig. 4-SM).

To give insight into the mechanism of photoreaction of irradiated GO, in the presence or absence of oxygen, we analyzed the soluble fraction obtained after filtration of the GO/rGO particles after different irradiation times. The soluble fraction was characterized by measuring the absorption and fluorescence UV-Vis absorption spectra and the TOC concentration.

Fig.5 shows the UV-Vis spectra of the filtered suspension of GO at different $t_{\text {irr }}$ both under air (Fig.5a) and $\mathrm{N}_{2}$ (Fig.5b). The main difference between the two cases is the dramatic increment of the absorbance with the complete photo-solubilization of GO under air after 14 days of irradiation (see the inset of Fig.5a). The spectra of the soluble fraction in each case show a roughly monotonic and exponential decrement with time of the absorbance in the UV region. The absorption spectra of the filtered suspensions of the samples under $\mathrm{N}_{2}$ differ from those under air. In this case the species released in solution showed a more structured spectra with a profile characterized by a maximum at $270 \mathrm{~nm}$ and a shoulder at $\approx 230 \mathrm{~nm}$. It is very hard to infer into the chemical nature of the photoreleased species, but the differences between the absorption spectra of the released soluble fractions in the presence or absence of oxygen suggest different operational mechanisms which give, in both cases, not only the partial reduction of the GO structure, but also the release in solution of different species.

The trend of TOC concentration under the two experimental conditions explored is reported in Fig.6. A bell-shaped behavior was observed in both cases in the first 7 days of irradiation. As expected, under oxygen after complete dissolution of $\mathrm{GO} / \mathrm{rGO}$ the TOC concentration increases till $\approx 9 \mathrm{mg} \mathrm{C} \mathrm{dm}^{-3}$. Note that the bell-shaped behavior for TOC suggests that the irradiation of GO not only activates a self-transformation/reduction process, but can also activate the phototransformation of low molecular organic molecules in solution, in this case directly released from the GO structure. This has suggested the potential use of irradiated GO as photo-activator for the degradation of organic molecules (e.g. pollutants) as reported below. 

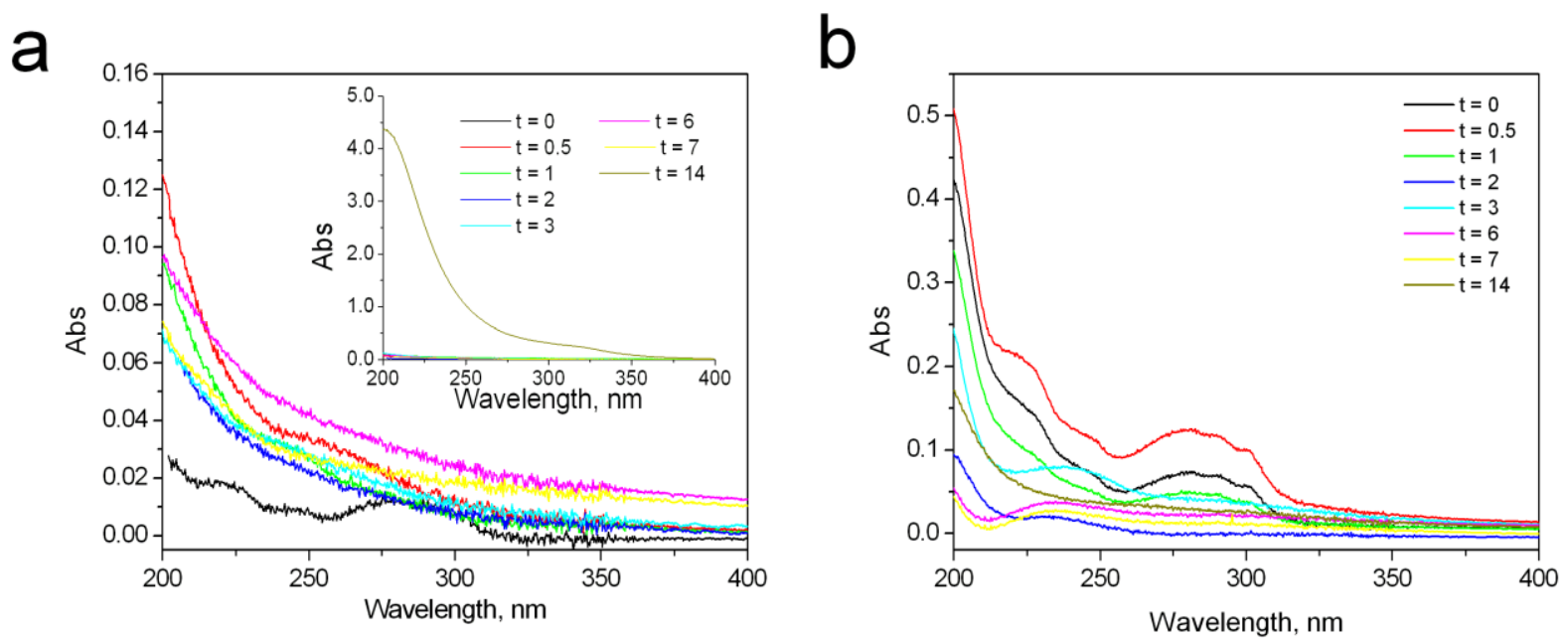

Fig.5 UV-Visible spectra of the $0.45 \mu \mathrm{m}$ filtered suspensions after different irradiation time under air (a, from 0 to 7 days) and under nitrogen (b, from 0 to 14 days). Inset of a: UV-Visible spectra of the filtered suspensions after irradiation under air from 0 to 14 days.

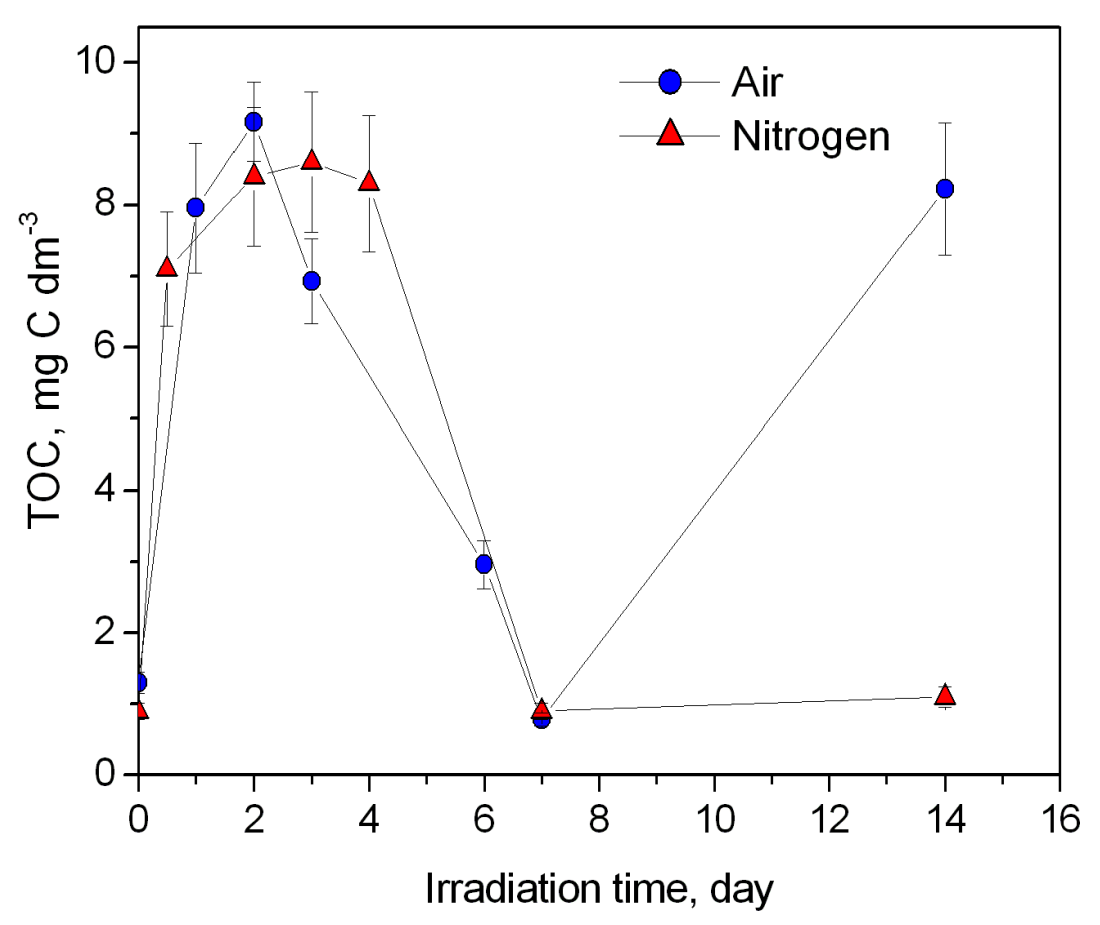

Fig.6 Total Organic Carbon concentration in the filtered GO suspensions $\left(30 \mathrm{mg} \mathrm{C} \mathrm{dm}^{-3}\right)$ subjected at different UV-VIS irradiation time under air (circles) and under nitrogen (triangles)

The EEMs of the filtered suspension subjected to UV-Vis irradiation for different times were recorded and reported in Fig. 7 (a: irradiation under air; b: irradiation under $\mathrm{N}_{2}$ ). In both cases we observed the formation of intense emission signal after irradiation as a consequence of the release in solution of fluorescent compounds. Under air the fluorescence peak is centered at Ex/Em coordinates $330 / 455 \mathrm{~nm}$. The position of this peak does not change with the increment of the 
irradiation time, while the intensity reaches a maximum after 2 days (note the perfect correspondence with the maximum of the TOC profile) and then decreases monotonically presumably as a consequence of the photo-activated degradation of the soluble compound released by the remaining GO/rGO. Similar Ex/Em coordinates of fluorescence have been reported by Coble [29] for samples of marine humic acid. This similarity suggests the presence in our sample of a complex mixture of compounds with moieties similar to those of natural humic substances (e.g. phenolic rings, carboxylic aliphatic/aromatic compounds...). Very interestingly, after 14 days of irradiation no fluorescence signal was recorded despite of the high value of TOC and high adsorption in the UV region. The photo-dissolution of $\mathrm{rGO}$ at prolonged irradiation time gives species characterized by scarce/null fluorescence, but high absorption coefficient in the UV region (presumably low molecular and highly hydrophilic compounds characterized by low degree of aromaticity). The EEMs recorded on the filtered samples obtained after irradiation under $\mathrm{N}_{2}$ (Fig. 7b) shows an overall behavior similar to that observed on the sample obtained under air. The fluorescence signal peaks after $2 / 3$ days according with the previously commented maximum of the TOC. The position of the fluorescence signals is different compared to that observed for the solution obtained in non-anoxic condition and shifts from 300/355 nm (after 0.5 days) to 320/430 $\mathrm{nm}$ (after 4 days). The decrement in the fluorescence intensity is totally ascribed to the decrease of the TOC concentration after the first 3 days of irradiation.

With the same approach we monitored the concentration of anions in the filtered solution at different irradiation times. The ion chromatograms obtained both under air and under $\mathrm{N}_{2}$ were reported in Fig.5-SM. The two main peaks observed are related to the presence of chloride and sulfate, residues of the adopted synthetic methods. Furthermore, only under air and at $t_{\text {irr }} \geq 7$ days did we observe a significant concentration of organic anions (mixture of organic carboxylates, e.g. acetate, formiate...) proof of the presence under air of a photo-activated mechanism able to give the dissolution of $\mathrm{rGO} / \mathrm{GO}$ with the formation of low MW compounds with oxygen-containing moieties such as carboxylic groups. In $\mathrm{N}_{2}$ the concentration of carboxylic compounds are always low, the only organic anion identified in traces is the acetate ion which is released in solution during the first irradiation days, presumably as a consequence of the GO photo-reduction process which releases in solution carboxylic moieties formed on the graphenic structure during the oxidation step. 

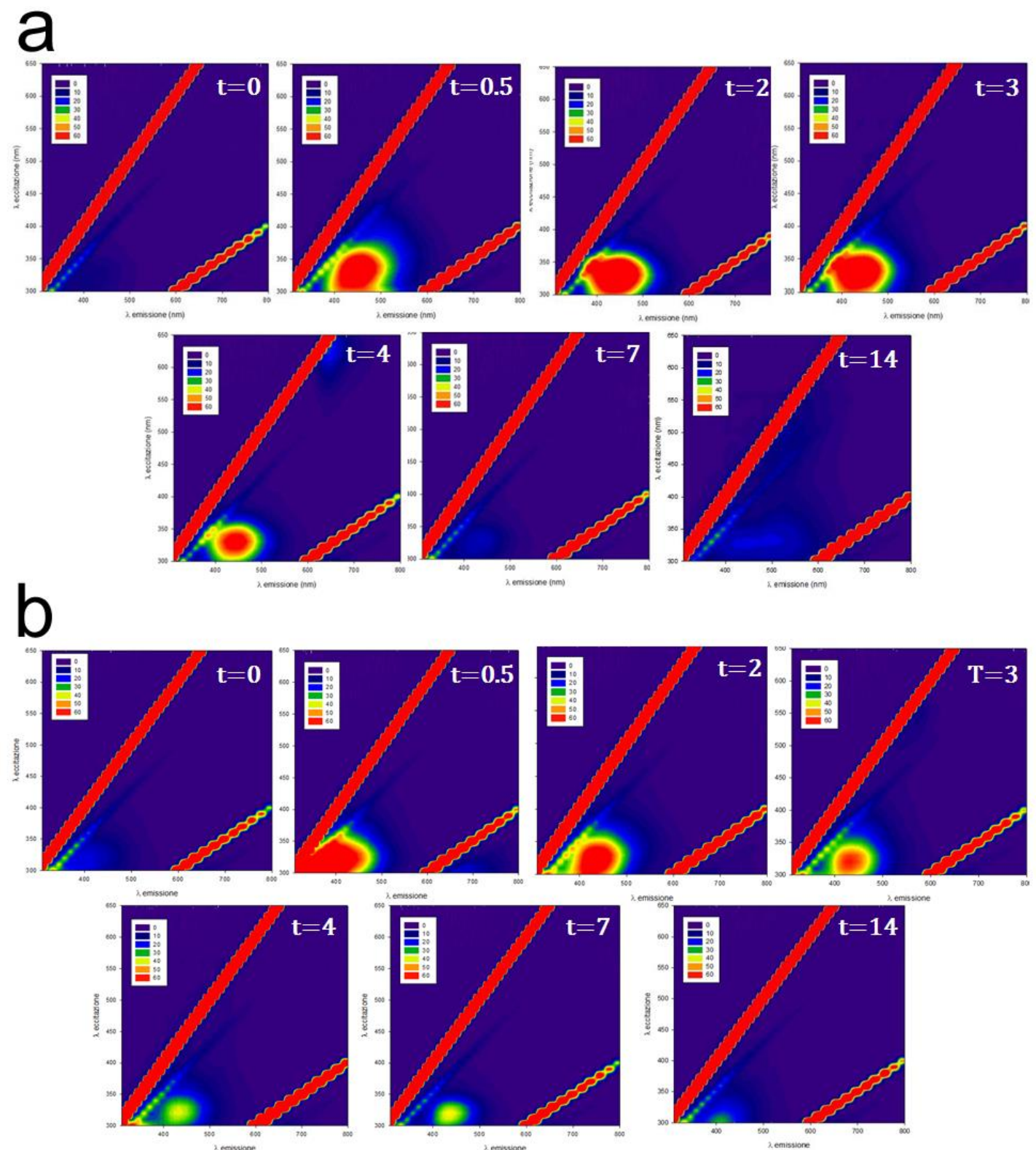

Fig. 7. Excitation/Emission matrixes (EEMs) of the filtered GO irradiated samples after different irradiation time under air (A) and under deoxygenated atmosphere (B)

The release of gaseous species as a consequence of the irradiation of film or suspension of GO was previously reported [26,28,30]. In particular Shulga and co-workers [28] observed the release of $\mathrm{CO}, \mathrm{CO}_{2}, \mathrm{H}_{2} \mathrm{O}$ and $\mathrm{O}_{2}$ during the irradiation of $\mathrm{GO}$ films, with a main production of $\mathrm{CO}$ in the first steps of irradiation and a progressive and continued accumulation of $\mathrm{CO}_{2}$. The authors reported a significant release of the same gases during the heating treatment of GO in the $150-230{ }^{\circ} \mathrm{C}$ range; in addition, traces of methane, formaldehyde and formic acid were observed. 

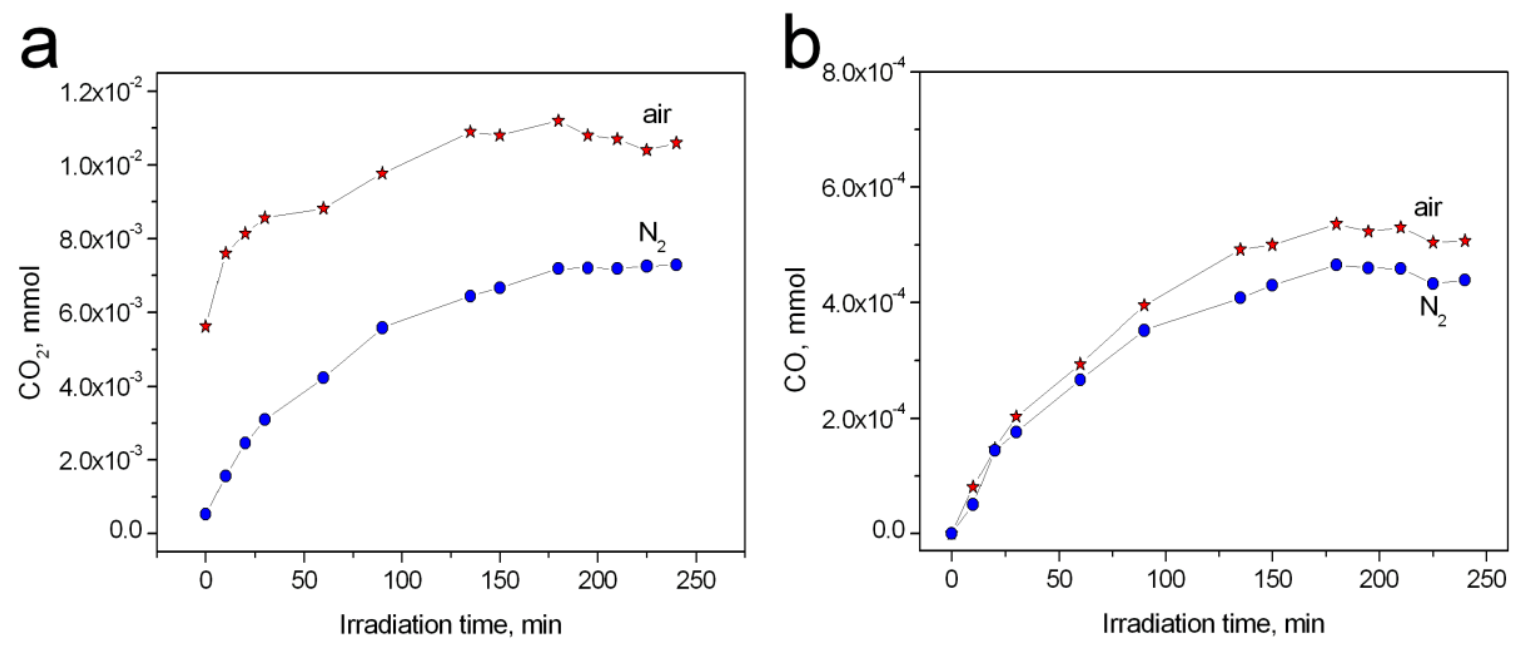

Fig.8 Carbon dioxide (a) and carbon monoxide (b) release from an irradiated GO water suspension (GO concentration $4000 \mathrm{mg} \mathrm{C} \mathrm{dm}^{-3}$ ).

Fig. 8 shows the trend of $\mathrm{CO}_{2}$ and $\mathrm{CO}$ in the headspace of the testing cells both under air and under $\mathrm{N}_{2}$. The main formed gases during the irradiation were $\mathrm{CO}_{2}$ and $\mathrm{CO}$, even if the carbon monoxide concentration is 10 times lower than that of $\mathrm{CO}_{2}$. For both gases a cumulative behavior was observed and no significant differences were observed under the two considered experimental conditions. The bias of $\mathrm{CO}_{2}$ before irradiation in the experiment under air is clearly due to the concentration of $\mathrm{CO}_{2}$ in the air of laboratory. Furthermore, traces of $\mathrm{CH}_{4}$ and $\mathrm{H}_{2}$ were observed. The absence of significant differences between the rate of gaseous release in the presence or absence of oxygen suggests the presence of an operational mechanism which gives the release of $\mathrm{CO}_{2}$ and/or $\mathrm{CO}$, and does not involve an active role of oxygen (e.g. a photoinduced decarboxylation /decarbonylation).

The characterization of the rGO particles obtained at different irradiation times under air and under anoxic atmosphere was carried out recording the ATR-FTIR spectra of the particles collected on the surface of the filtering membranes. Spectrum recording was not easy because the collected rGO (see Fig.6-SM) had very high absorbing coefficient in the whole range of wavelengths, which hinders a sufficient propagation of the evanescent wave in the ATR cell. As a consequence the high quality of the spectra obtained on the pristine GO (see Fig.3) cannot be obtained on the rGO samples. The ATR-FTIR spectra of the rGO samples obtained under irradiation under air and under $\mathrm{N}_{2}$ are reported in Fig.7-SM A and B, respectively. The spectra of the samples change as expected with the irradiation time both under air and under $\mathrm{N}_{2}$ and an overall decrement of the oxidation degree of the original material was observed. Particularly, a net shift of the signal at $1620 \mathrm{~cm}^{-1}$ towards lower wavenumbers with the increment of the irradiation time is consistent with an increase 
of the aromaticity of the structure; furthermore, the disappearance of the signal at $\approx 990 \mathrm{~cm}^{-1}$ is related to the loss of the epoxy and ether groups from the structure. However, the persistence of some oxidized moieties such as the $\mathrm{C}=\mathrm{O}$ groups $\left(\approx 1720 \mathrm{~cm}^{-1}\right)$ and hydroxyls can be emphasized, as a proof of the not complete photochemical reductive process. This is in agreement with the data reported in literature, in which a total recover of the original structures has never been reported [see 26 and references therein].

\section{GO sensitized phenol photo-transformation under UV and Vis irradiation}

The TOC concentration profiles reported in Fig.6 emphasize the ability of rGO to activate phototransformation processes able to mineralize the organic molecules released during the irradiation process of GO. This process seems to be more effective in the presence of oxygen. For this reason we investigated in this condition the phototrasformation ability of the irradiated GO toward a model organic substrate, e.g. phenol.

Adsorption experiments in the dark showed that phenol was poorly adsorbed on GO. Direct photolysis scarcely contributed to the phenol transformation, too; upon light exposure in pure water its degradation was negligible even after long irradiation times.

As previously reported, graphene oxide exhibits a certain photoactivity when dispersed in a polymeric film [31]. Similarly, when dispersed in aqueous solution it induced phenol degradation, as assessed in Fig. 9 where the phenol initial kinetic constants as a function of GO concentration are shown. The highest degradation rate was obtained at very low GO concentration $\left(100 \mathrm{mgL}^{-1}\right)$, where the complete disappearance of phenol is obtained within $3 \mathrm{~h}$ of irradiation. With increasing GO concentration, we observed a decrease in both the initial rate of disappearance and in the percentage of phenol abatement. This could be justified considering the deep color of the suspension which shields the incoming light. The observed rate $\left(r_{o b s}\right)$ in the experimental system is given by equation (1)

$$
r_{o b s}=\int_{0}^{b} r(x)
$$

where $b$ is the optical length of the cell, $r(\mathrm{x})$ is the reaction rate for the optical path $x$ and depends on the light intensity that reaches the portion $x$. For a photochemical process occurring in a solution that absorbs, there is a concentration quenching on the overall rate; for a photocatalytic system, a rigorous model was reported elsewhere [32]. 


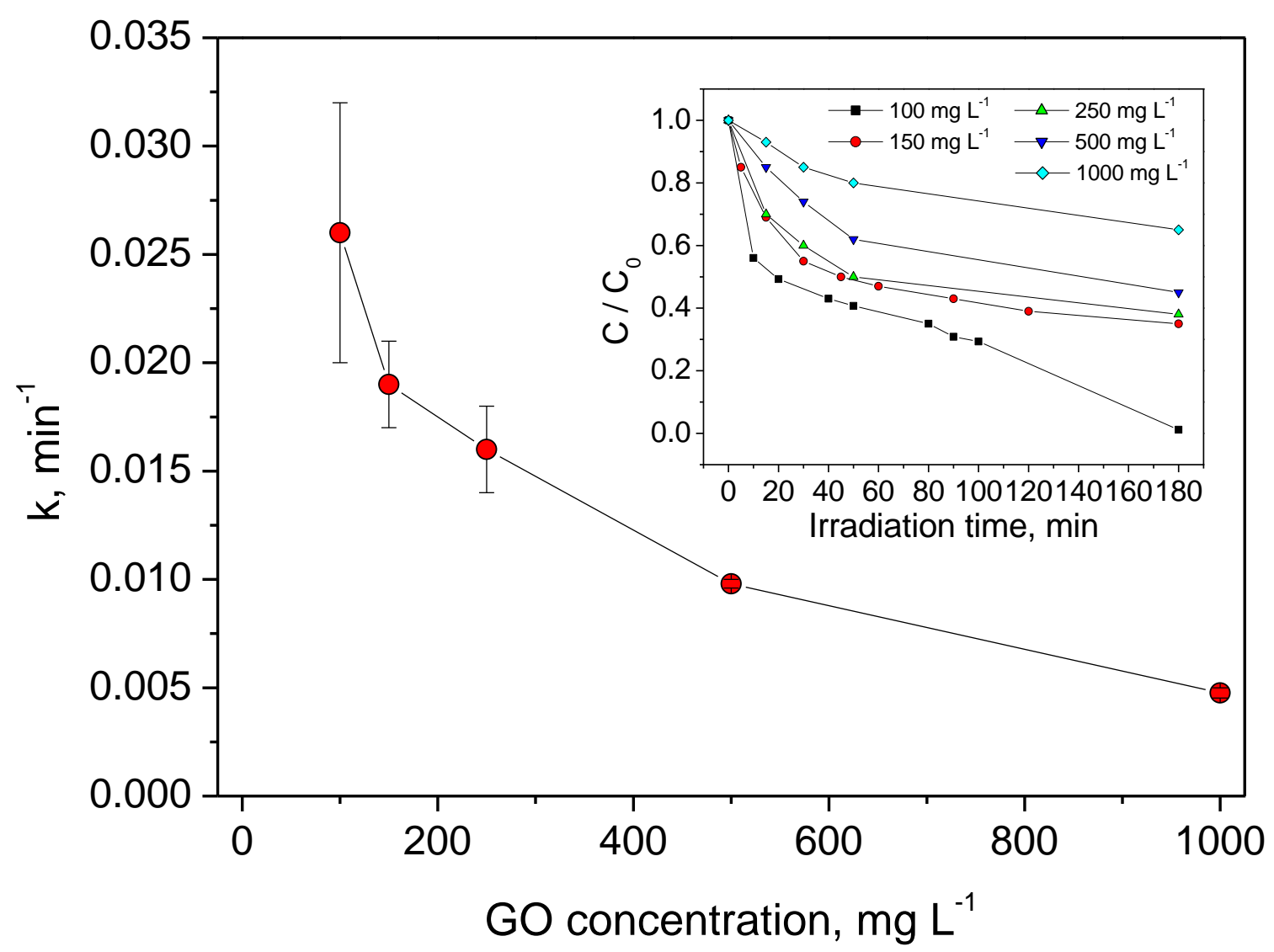

Fig.9 First order degradation constant $\left(\mathrm{min}^{-1}\right)$ for the phenol degradation process as a function of the GO concentration. Inset: Phenol degradation over time irradiation in the presence of different GO concentrations.

However, in all disappearance curves a double kinetics can be evidenced. Initially, rate is high and pseudo-first order kinetics are followed; then, in the second tract, degradation becomes slower. These data could be justified by assuming that GO behaves as a reagent, starting a photochemical (and not a photocatalytic) process. The support to this hypothesis came from experiments in which we studied the phenol degradation at very low GO concentration by changing the phenol (P) to GO ratio (see Fig. 10). Interestingly, triple kinetics is clearly evidenced with both P:GO ratio of 1:1 or with a GO defect (P:GO=2:1). 


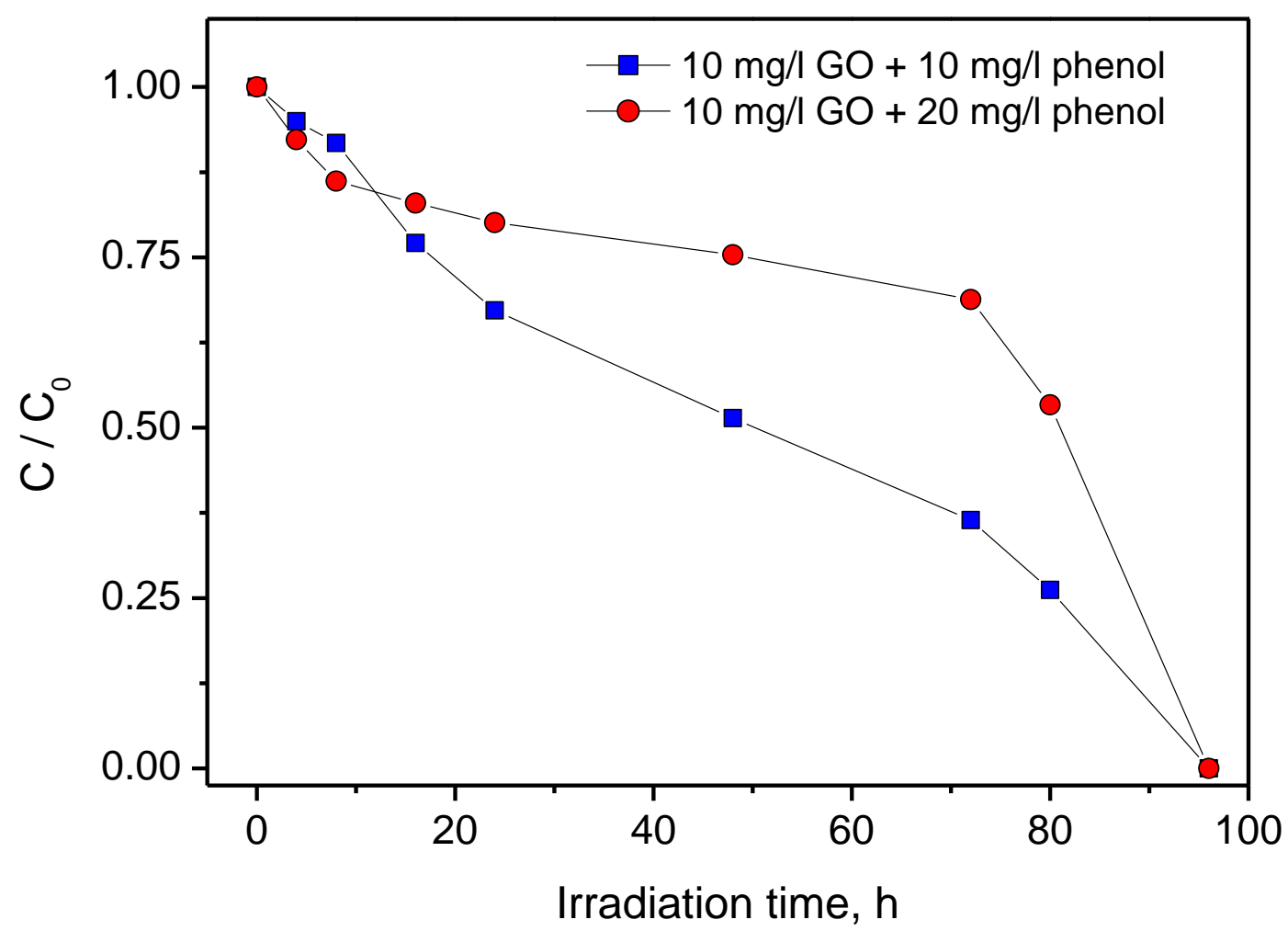

Fig.10 Phenol degradation over time irradiation at low GO concentrations.

In the first tract, the degradation of $20 \%$ (ratio $2: 1$ ) or $40 \%$ (ratio $1: 1$ ) of phenol occurs within few minutes, that is approximatively $4 \mathrm{ppm}$ of phenol degraded in both cases in the presence of $10 \mathrm{ppm}$ GO. Then, reaction slows down and levels off at almost $25 \%$ or $50 \%$ of $\mathrm{P}$ abated, respectively, until $72 \mathrm{~h}$ of irradiation. In particular, from data with $20 \mathrm{ppm}$ phenol until $60 \mathrm{~h}$, the curves could be well fitted by a $2^{\text {nd }}$ order kinetic in which:

Phenol $+\mathrm{GO} \rightarrow$ products

where GO is the limiting reagent. This shape supports the photochemical and not the photocatalytic role of GO. Later, in both cases a sharp increase in the disappearance rate is observed and complete phenol abatement is achieved within $96 \mathrm{~h}$. This $3^{\text {rd }}$ reaction region could be interpreted taking into account the bell-shaped TOC curve described in fig. 6. During the first 2 days of GO irradiation, a sharp TOC increase was observed, assessing that a competition between GO selftransformation/reduction and phenol degradation exists and leads to a lowered degradation efficiency. After $72 \mathrm{~h}$ of GO irradiation, a sharp TOC decrease occurs, attributable to the formation of species with a marked photoactivity which permit not only to degrade the organic small molecules formed from GO, but also phenol. 


\section{Conclusions}

The photo-reduction of GO to rGO occurs both under air and under anoxic condition. Oxidized moieties, such as hydroxyls and carbonyl groups, remain in the studied materials even after few days of irradiation. The mechanisms of photo-reduction under air and under $\mathrm{N}_{2}$ lead to similar materials, but are different considering the dissimilar spectroscopic properties of the soluble molecules released in solution. No difference in the nature and amount of gaseous species released were observed in the two experimental conditions considered.

The obtained rGO shows a marked photoactivity. The rGO photo-degradation ability was observed not only toward the compounds released during the first irradiation period, but also when irradiating phenol in the presence of GO, so underlining the GO ability to act as photoactivator. This photoactivity in oxygen forces the photo-degradation of the rGO structure itself till a complete dissolution via an oxidative mechanism (presumably activated by the excited state of the rGO particles). In nitrogen the step of self photo-degradation of rGO is hindered / depressed or proceeds with very slow kinetics increasing the photostability of the hydrophobic rGO particles.

\section{Acknowledgements}

This research is supported by a Marie Curie International Research Staff Exchange Scheme Fellowship (PHOTOMAT, proposal n. 318899) within the $7^{\text {th }}$ European Community Framework Programme. MM and MD are kind grateful to Dr. Chiara Deiana for her help during the ATR-FTIR measures.

\section{References}

[1] Brodie BC (1859) On the Atomic Weight of Graphite. Philos Trans R Soc 149:249-259.

[2] Staudenmaier L (1898) Method of preparation of graphite-acid, Berichte der Deutschen Chemishen Gesellschaft 31:1481-1499.

[3] Hummers WS and Offeman RE (1958) Preparation of graphitic oxide. J Am Chem Soc 80:1339.

[4] Wang Y, Chen X, Zhong Y, Zhu F, Loh KP, Dreyer DR, Park S, Bielawski CW and Ruoff RS (2010) The chemistry of graphene oxide, Chem Soc Rev 39:228-240.

[5] Compton OC, Binh S and Nguyen T (2010) Graphene Oxide, Highly Reduced Graphene Oxide, and Graphene: Versatile Building Blocks for Carbon-Based Materials, Small 6:711-723. 
[6] Krishnamoorthy K, Veerapandian M, Yun K, Kim SJ (2013) The chemical and structural analysis of graphene oxide with different degrees of oxidation, Carbon 53: 38-49

[7] Mathkar A, Tozier D, Cox P, Ong P, Galande C, Balakrishnan K, Leela A and Ajayan P (2012) Controlled, Stepwise Reduction and Band Gap Manipulation of Graphene Oxide, J Phys Chem Lett 3:986-990.

[8] Williams G, Seger B and Kamat PV (2008) $\mathrm{TiO}_{2}$-graphene nanocomposites. UV-assisted photocatalytic reduction of graphene oxide, ACS Nano 2:1487-1491.

[9] Shah MdSAS, Park AR, Zhang K, Park JH and Yoo PJ (2012) Green Synthesis of Biphasic $\mathrm{TiO}_{2}$-Reduced Graphene Oxide Nanocomposites with Highly Enhanced Photocatalytic Activity, ACS Appl Mater Interfaces 4:3893-3900.

[10] Stengl V, Popelkova D and Vlacil P (2011) $\mathrm{TiO}_{2}-$ Graphene Nanocomposite as High Performace Photocatalysts, J Phys Chem C 115:25209-25219.

[11] Du J, Lai X, Yang N, Zhai J, Kisailus D, Su F, Wang D and Jiang L (2011) Hierarchically Ordered Macro-Mesoporous $\mathrm{TiO}_{2}-$ Graphene Composite Films: Improved Mass Transfer, Reduced Charge Recombination, and Their Enhanced Photocatalytic Activities, ACS Nano 5:590-596.

[12] Long M, Qin Y, Chen C, Guo X, Tan B and Cai W (2013) Origin of Visible Light Photoactivity of Reduced Graphene Oxide/ $/ \mathrm{TiO}_{2}$ by in Situ Hydrothermal Growth of Undergrown $\mathrm{TiO}_{2}$ with Graphene Oxide, J Phys Chem C 117:16734-16741.

[13] Jiang B, Tian C, Zhou W, Wang J, Xie Y, Pan Q, Ren Z, Dong Y, Fu D, Han J and Fu H (2011) In Situ Growth of $\mathrm{TiO}_{2}$ in Interlayers of Expanded Graphite for the Fabrication of $\mathrm{TiO}_{2}$ Graphene with Enhanced Photocatalytic Activity, Chem Eur J 17:8379-8387.

[14] Sun L, Zhao Z, Zhou Y and Liu L (2012) Anatase $\mathrm{TiO}_{2}$ nanocrystals with exposed $\{001\}$ facets on graphene sheets via molecular grafting for enhanced photocatalytic activity Nanoscale 4:613-620.

[15] Huang NM, Lim HN, Chia CH, Yarmo MA and Muhamad MR (2011) Simple roomtemperature preparation of high-yield large-area graphene oxide, Int J Nanomed 6:3443-3448.

[16] Mei Q, Zhang K, Guan G, Liu B, Wang S and Zhang Z (2010) Highly efficient photoluminescent graphene oxide with tunable surface properties, Chem Commun 46:7319-7321.

[17] Li D, Mueller MB, Gilje S, Kaner RB and Wallace GG (2008) Processable Aqueous Dispersions of Graphene Nanosheets, Nat Nanotechnol 3:101-105.

[18] Marcano DC, Kosynkin DV, Berlin JM, Sinitskii A, Sun Z, Slesarev A, Alemany LB, Lu W and Tour JM (2010) Improved Synthesis of Graphene Oxide, ACS Nano 4:4806-4814. 
[19] Gao X, Jang J and Nagase S (2010) Hydrazine and Thermal Reduction of Graphene Oxide: Reaction Mechanisms, Product Structures, and Reaction Design, J Phys Chem C 114:832-842. [20] Shang JI, Ma L, Li J, Ai W, Yu T and Gurzadyan G (2012) The origin of fluorescence from graphene oxide, Sci Rep 2:1-8.

[21] Galande C, Mohite AD, Naumov AV, Gao W, Ci L, Ajayan A, Gao H, Srivastava A, Weisman RB and Ajayan PM (2011) Quasi-molecular fluorescence from graphene oxide, Sci Rep, 85:1-5.

[22] Li J-L, Tang B, Yuan B, Sun L and Wang X-G (2013) A review of optical imaging and therapy using nanosized graphene and graphene oxide, Biomaterials 34:9519-9534.

[23] L. Cao, M. J. Meziani, S. Sahu and Y. P. Sun (2012) Photoluminescence Properties of Graphene versus Other Carbon Nanomaterials, Accounts Chem Res 46:171-180.

[24] Park J, Lee WH, Huh S, Sim SH, Kim SB, Cho K, Hong BH and Kim KS (2011) WorkFunction Engineering of Graphene Electrodes by Self-Assembled Monolayers for HighPerformance Organic Field-Effect Transistors, J Phys Chem Lett 2:841-845.

[25] Park J, Jo SB, Yu YJ, Kim Y, Yang JW, Lee WH, Kim HH, Hong BH, Kim P, Cho K and Kim KS (2012) Single-Gate Bandgap Opening of Bilayer Graphene by Dual Molecular Doping, Adv Mater 24:407-411.

[26] Guardia L, Villar-Rodil S, Paredes JI, Rozada R, Martınez-Alonso A and Tascón JMD (2012) UV light exposure of aqueous graphene oxide suspensions to promote their direct reduction, formation of graphene-metal nanoparticle hybrids and dye degradation, Carbon 50:1014-1024.

[27] Fernández-Merino MJ, Guardia L, Paredes JI, Villar-Rodil S, Solís-Fernández P, MartínezAlonso A and Tascón JM (2010) Vitamin C Is an Ideal Substitute for Hydrazine in the Reduction of Graphene Oxide Suspensions, J Phys Chem C 114:6426-32.

[28] Shulga YM, Martynenko VM, Muradyan VE, Baskakov SA, Smirnov VA and Gutsevl GL (2010) Gaseous products of thermo- and photo-reduction of graphite oxide, Chem Phys Lett 498: 287-291.

[29] Coble PG (1996) Characterization of marine and terrestrial DOM in seawater using excitationemission matrix spectroscopy, Mar Chem 51:325-346.

[30] Plotnikov VG, Smirnov VA, Alfimov MV and Shul'ga YM (2011) The graphite oxide photoreduction mechanism, High Energy Chem 45:411-415.

[31] Sangermano M, Calza P and Lopez-Manchado MA (2013) Graphene oxide-Epoxy hybrid material as innovative photocatalyst, J Mater Sci 48:5204-5208.

[32] Minero C and Vione D (2006) A quantitative evaluation of the photocatalytic performance of $\mathrm{TiO}_{2}$ slurries, Appl Catal B-Environ 67:257-269. 